- RAHIS, Revista de Administração Hospitalat e Inovação em Saúde Vol. I7, nI " Belo Horizonte, MG "JAN/MAR 2020 "e-ISSN: 2177 2754 e ISSN impresso: 1983-5205 " DOI: https://doiorg/I0.21450/rahis.vI7iI.6198 " Submetido: (08/05/2020) " Aceito: (21/05/2020) " Sistema de avaliação: Double Blind Review "p. I - 20.

\title{
A CONFIANÇA DE ENFERMEIROS NA ORGANIZAÇÃO HOSPITALAR
}

THE TRUST OF NURSES IN THE HOSPITAL ORGANIZATION

\section{LA CONFIANZA DE LAS ENFERMERAS EN LA ORGANIZACIÓN HOSPITALARIA}

\author{
Marcio Pedroso Juliani \\ Faculdade Anhanguera, Unidade Passo Fundo - RS \\ marpedro66@yahoo.com.br \\ Priscila Cerutti \\ Faculdade Anhanguera, Unidade Passo Fundo - RS \\ priscilacerutti@yahoo.com.br \\ Cintia Sonale Rebonatto \\ Faculdade Meridional - IMED \\ cintiasonale@gmail.com \\ José Carlos Zanelli \\ jczanelli@terra.com.br \\ Universidade Federal de Santa Catarina - UFSC
}




\section{RESUMO}

Relações de confiança têm sido consideradas fundamentais para o comprometimento com os resultados organizacionais. Assim, este estudo teve como objetivo analisar a relação de confiança entre os enfermeiros e o hospital em que trabalham. Para tanto, realizou-se uma pesquisa quantitativa-qualitativa com 58 enfermeiros que compõem o quadro de empregados de um hospital localizado no norte do Rio Grande do Sul, através da aplicação de questionário e de complementação de sentenças elaborados a partir da Escala de Confiança do Empregado na Organização. Os resultados mostraram que os fatores mais confiáveis para os enfermeiros são os padrões éticos, seguidos pela solidez organizacional.

Palavras-chave: Confiança. Enfermeiros. Hospital.

\section{ABSTRACT}

Trust relationships have been considered fundamental to the commitment of the organizational results. Thus, this study aimed to analyze the relationship of trust between the nurses and the hospital they work for. Thereby, a quantitative-qualitative research was conducted with 58 nurses who make up the staff of a hospital located in north Rio Grande do Sul, through the application of a questionnaire and a complementation of sentences, elaborated from the Confidence Scale of the Employee in the Organization. The results showed that the most reliable factors for the nurses are the ethical standards, followed by the organizational solidity.

Keywords: Trust. Nurses. Hospital.

\section{RESUMEN}

Las relaciones de confianza se han considerado fundamentales para el compromiso con los resultados organizacionales. Entonces, este estudio tuvo como objetivo analizar la relación de confianza entre las enfermeras y el hospital con el que trabajan. Para ello, se realizó una investigación cuantitativa-cualitativa con 58 enfermeras que integran el personal de un hospital ubicado en el norte de Rio Grande do Sul, mediante la aplicación de un cuestionario y la complementación de oraciones elaboradas a partir de la Escala de confianza empleada en la Organización. Los resultados mostraron que los factores más confiables para las enfermeras son, principalmente, los estándares éticos, seguidos de la solidez organizacional.

Palabras-clave: Confianza. Enfermeras. Hospital.

\section{INTRODUÇÃO}

Relações de confiança têm sido consideradas básicas para o comprometimento com o desenvolvimento e os resultados do trabalho nas organizações (ANDRADE, et al., 2018). Os níveis de confiança dos trabalhadores estão fortemente relacionados à percepção de transparência, de integridade e de consistência nas relações de trabalho (ZANINI, 2007), bem como de justiça (SAMPAIO; GOMIDE Jr; OLIVEIRA, 20I9) e de ética organizacional (CUNHA; SILVA; ESTIVALETE; HÖRBE, 20I7). Portanto, o entendimento de como a confiança se apresenta nas organizações, ou mesmo a identificação de sua ausência, configura-se um aspecto importante para compreender as mudanças que o contexto organizacional enfrenta.

O risco inerente às mudanças organizacionais faz da confiança uma variável central para compreender como os empregados percebem tais modificações, bem como seus sentimentos para com a organização em que estão inseridos (CUNHA et al., 2017; NEVES; CAETANO, 2006). Desse modo, a confiança reduz a complexidade social e garante mais segurança para as escolhas que se fazem necessárias (FISHER; NOVELLI, 2008), além de contribuir para a redução da sensação de vulnerabilidade dos 
empregados, favorecendo a capacidade de ação e estimulando a contribuição para o processo de trabalho cooperativo (FISHER; NOVELLI, 2008).

Nesse sentido, a confiança organizacional desempenha um papel crítico, no sentido de estar ligada à preservação ou à ruptura do relacionamento entre o empregado e a organização em que trabalha (NIENABER; HOFEDITZ; SEARLE, 20I4). Considerada uma variável de crescente interesse para investigações referentes ao comportamento organizacional e outras áreas correspondentes, a confiança organizacional tem sido objeto de diversas pesquisas, sendo relacionada ao clima organizacional (CHIUZI; SIQUEIRA; MARTINS, 20I2; DIAS; OLIVEIRA, 20I6), à efetividade organizacional, à inteligência emocional (BARROS, 20II), ao bem-estar e à qualidade de vida (SILVA; OLIVEIRA, 20I7; LUIS; RODRÍGUEZ; GÓMEZ, 2020), ao comprometimento organizacional (CUNHA; SILVA; ESTIVALETE; HÖRBE, 2017), aos valores pessoais e organizacionais (OLIVEIRA; SOUZA, 20I4) e à performance (VERBURG et al., 2018).

Diante disso, estudar a confiança em organizações vem adquirindo crescente importância na literatura, uma vez que a compreensão do fenômeno confiança entre pessoas, grupos ou organizações fornece evidências para abordagens em diversos campos, como sociologia, economia, psicologia, enfermagem, administração, dentre outras (TZAFRIR; HAREL, 2002; DESMET; CREMER; VAN DIJK, 20II). E, nesse sentido, torna-se uma temática de destaque nas discussões em todos os setores da sociedade (LOURENÇO et al., 20II). A importância da confiança, em termos dos vários níveis organizacionais, tem sido cada vez mais reconhecida (FULMER; GELFAND, 20I5), independentemente do ramo em que a organização atua. Esse fato não é diferente nas instituições prestadoras de cuidados de saúde, como os hospitais, que devem eleger a confiança organizacional como um foco estratégico em suas atividades (LOURENÇO et al., 20I I).

Para tanto, quando buscado acerca das pesquisas que relacionam a confiança no contexto hospitalar, a maior parte dos estudos refletem acerca de sua relação com o stress de enfermeiros (TOP; TEKINGUNDUZ, 20I9), o engajamento (AKGUNDUZ; GUZEL, 20I4), a intenção de abandonar o emprego (OZYILMAZ, 20I0), mas, principalmente a confiança é estudada nas relações do cuidado entre enfermeiro-paciente (LOURENÇO et al., 20II; OZARAS; ABAAN, 20I6; BAPTISTA et al., 20I8). No entanto, são escassos os estudos que tenham relacionado a confiança organizacional especificamente de enfermeiros, avaliados a partir dos cinco fatores da confiança encontrados na Escala de Confiança Organizacional do Empregado (ECOE), a saber: (I) promoção do crescimento do empregado; (2) solidez organizacional; (3) normas relativas à demissão do empregado; (4) reconhecimento financeiro organizacional; e, (5) padrões ético.

A motivação do estudo recai em compreender empiricamente as interações entre os cinco construtos que fazem parte da ECEO nos profissionais enfermeiros que atuam em um hospital localizado na região norte do estado do Rio grande do Sul. A análise dessas temáticas sob a perspectiva dos trabalhadores do setor hospitalar enriquece o panorama sobre as interações entre os referidos construtos, uma vez que a área da saúde contribui sobremaneira para as mudanças ocorridas na sociedade. Dessa forma, o objetivo deste estudo consiste em analisar as relações existentes entre a confiança organizacional de enfermeiros no hospital que atuam.

\section{CONFIANÇA ORGANIZACIONAL}

As organizações são formadas, de forma geral, por estruturas burocráticas e hierárquicas, sendo que a confiança se torna um mecanismo informal para coordenar e controlar as rotinas organizacionais (ZANINI, 2007). Quando existe confiança nas relações burocráticas, as organizações expandem de forma significativa seu potencial de relacionamentos cooperativos, bem como sua eficiência gerencial (ZANINI, 2007; LUIS; RODRÍGUEZ; GÓMEZ).

Apesar de ter sua relevância reconhecida, pesquisadores frisam não haver consenso quanto a sua conceituação (CHHETRI, 20I4). Assim, ela é definida como um conjunto de crenças a respeito de 
padrões éticos da organização, nas quais ocorre o uso limitado do oportunismo em suas relações e da competência que se traduz em sua capacidade técnica e solidez financeira (IANAGUIVARA, 20I I). Já, outros autores tangenciam seus estudos tendo como foco de análise os aspectos da organização percebidos pelos empregados, não direcionando o seu interesse sobre a confiança na perspectiva das relações sociais (DIAS; OLIVEIRA, 20I6).

Corroborando, Maguire e Phillips (2008) conceituam confiança organizacional como a expectativa de um indivíduo de que o sistema organizacional agirá com previsibilidade e boa vontade. Dessa forma, quando se olha para a confiança organizacional, o referente de confiança é um coletivo, ou sistema, incluindo múltiplos atores ao invés de um único indivíduo ou grupo específico (VERBURG et al., 2018). Para Tan e Lim (2009), a confiança na organização está relacionada com a disposição dos empregados de ficarem indefesos contra as ações e o comportamento organizacional, que eles não podem controlar. E, ainda, Williams (2005) complementa que a confiança organizacional envolve as expectativas positivas que os empregados têm sobre as intenções e ações, baseadas em papéis, relacionamentos, experiências e suas interdependências dentro da organização.

A confiança organizacional deriva das avaliações dos empregados quando se questionam se a organização tem competência para cumprir metas e responsabilidades de forma confiável (capacidade organizacional); quando sinaliza intenções positivas em relação ao bem-estar das partes interessadas (benevolência organizacional); e quando adere aos princípios morais geralmente aceitos (integridade organizacional) em suas relações com diferentes stakeholders (GILLESPIE; DIETZ, 2009; SEARLE et al., 20II).

No intuito de medir a confiança do empregado na organização, Oliveira e Tamayo (2008) construíram e validaram a Escala de Confiança do Empregado na Organização (ECEO), que visa avaliar a percepção dos empregados quanto: (I) à promoção do crescimento do empregado; (2) à solidez organizacional; (3) às normas relativas à demissão do empregado; (4) ao reconhecimento financeiro organizacional; e, (5) aos padrões éticos (Quadro I).

Quadro I - Definição dos fatores avaliados na confiança do empregado na ECEO

\begin{tabular}{|l|l|}
\hline $\begin{array}{l}\text { Antecedentes } \\
\text { (Fatores) }\end{array}$ & Definição \\
\hline $\begin{array}{l}\text { Promoção do } \\
\text { crescimento do } \\
\text { empregado }\end{array}$ & $\begin{array}{l}\text { O fator crescimento e promoção do empregado na organização está relacionado com o "incentivo ao } \\
\text { crescimento profissional dos empregados por meio de alternativas concretas e reais propostas pela } \\
\text { organização, permitindo aos colaboradores prever seu crescimento naquele contexto de trabalho." }\end{array}$ \\
\hline $\begin{array}{l}\text { Solidez } \\
\text { organizacional }\end{array}$ & $\begin{array}{l}\text { Refere-se à firmeza, à estabilidade financeira da organização cujos reflexos são o cumprimento de suas } \\
\text { obrigações financeiras com os empregados, como o pagamento de salário pontualmente, a perspectiva } \\
\text { de um futuro próspero, além da a capacidade de superar crises econômicas ocasionadas por planos } \\
\text { governamentais e mudanças de mercado }\end{array}$ \\
\hline $\begin{array}{l}\text { Normas relativas } \\
\text { à demissão do } \\
\text { empregado }\end{array}$ & $\begin{array}{l}\text { Refere-se a normas e procedimentos organizacionais, utilizados ou não, na demissão de empregados. } \\
\text { As demissões podem ocorrer considerando normas e procedimentos objetivos conhecidos pelos } \\
\text { empregados ou em função de julgamentos pessoais de dirigentes. }\end{array}$ \\
\hline $\begin{array}{l}\text { Reconhecimento } \\
\text { financeiro } \\
\text { organizacional }\end{array}$ & $\begin{array}{l}\text { Os esforços do empregado são reconhecidos e valorizados de forma financeira, principalmente por } \\
\text { meio do salário. A concessão de aumento salarial é percebida como uma das manifestações de } \\
\text { reconhecimento por parte da organização. Esse reconhecimento financeiro beneficia ambas as partes. }\end{array}$ \\
\hline Padrões éticos & $\begin{array}{l}\text { Princípios éticos como honestidade, igualdade, transparência da organização ao divulgar informações, } \\
\text { responsabilidade, manutenção de compromissos e respeito. Tais princípios orientam a relação da } \\
\text { organização com seus clientes e empregados ao procurar não prejudicar aqueles com os quais se } \\
\text { relaciona. }\end{array}$ \\
\hline
\end{tabular}

Fonte: Elaborado a partir de Oliveira (2004).

Nesse sentido, Oliveira e Tamayo (2008) compreendem a confiança organizacional como as crenças a respeito de padrões éticos, credibilidade da comunicação, poder econômico da organização e sua capacidade de reconhecer o desempenho do empregado, tanto financeira quanto profissionalmente. Assim, o empregado leva em conta a solidez econômica da organização, sua capacidade de honrar compromissos, reconhecer esforços individuais, além dos padrões morais e éticos da organização. 
Portanto, a confiança organizacional está relacionada ao comportamento recíproco que pode ou não ocorrer entre os indivíduos em uma organização (SOLOMON; FLORES, 2002), de forma que cumpram seus papéis de modo adequado ao bom funcionamento das relações e contribuam para a melhor qualidade de vida e maior produtividade organizacional (RUEDA; SERENINI; MEIRELES, 20I4).

Níveis mais baixos de confiança nas organizações fazem com que os empregados demonstrem menos criatividade, façam menos esforço para os propósitos da organização, tenham um baixo nível de moral, sejam menos produtivos e sintam-se estressados (RHEE, 2010). Por outro lado, níveis mais altos de confiança levam a índices mais elevados de produtividade, moral, desempenho organizacional, satisfação no trabalho, comprometimento, justiça organizacional e comportamento de cidadania, além da diminuição do absentéísmo (BOE, 2002).

Nesse sentido, compreende-se que a confiança tem um efeito direto nos resultados organizacionais (TAN; TAN, 2000). O estudo de Kim, Wang e Chen (2016) examinou a relação da confiança mútua entre 247 pares de supervisores-empregados para avaliar se esta relação estava associada a resultados no trabalho. Os achados mostraram um efeito positivo da confiança mútua percebida no desempenho da tarefa e na facilitação interpessoal. Assim, o desempenho das tarefas e a facilitação interpessoal aumentaram conforme a confiança entre supervisor e empregado também aumentavam.

Outro estudo avaliou o papel mediador e moderador da confiança organizacional na relação entre justiça e predisposição para atitudes retaliatórias em I 88 empregados do setor metalmecânico no Sul do Brasil (GOER GEN; PAULI; CERUTTI; PERIN, 20I9). Os resultados mostraram um efeito mediador indireto da confiança organizacional sobre a justiça e predisposição para atitudes de retaliação dos empregados, assim como o efeito moderador da confiança na a relação entre a justiça e a atitude de retaliação dos empregados também foi confirmada. Os resultados sugeriram que a confiança dos empregados na organização tem efeito mediador e moderador sobre a relação entre a justiça e a atitude de retaliação. Na mesma perspectiva, um estudo na Índia descobriu que a confiança organizacional está relacionada à justiça organizacional e ao desempenho no trabalho, e que a confiança organizacional aumenta se os empregados perceberem que a justiça organizacional é adequada (DECONINCK, 2010).

A pesquisa de Horta, Demo e Roure (20I2) investigou a confiança organizacional dos empregados e a relação com o bem-estar em I4I empregados de uma multinacional de tecnologia da informação. Os resultamos mostraram a existência de correlações positivas e significativas entre as duas variáveis, com associações que variaram de moderada $(>0,3$ e $<0,5)$ a forte $(>0,5)$. O estudo concluiu que a confiança está diretamente relacionada ao bem-estar, uma vez que possibilita condições de segurança e estabilidade na relação empregado-empresa.

Já no contexto hospitalar, enfermeiros com alta confiança organizacional possuem maior responsabilidade pelo atendimento ao paciente e fazem contribuições importantes para a realização dos objetivos do hospital (BASIT; DUYGULU, 2018). Assim, quando enfermeiros possuem confiança na organização que atuam, eles têm um aumento na percepção de justiça (ISCAN; SAYIN, 20I0), na satisfação profissional (VELEZ; STROM, 20I2), no engajamento (AKGUNDUZ; GUZEL, 20I4), diminuem o sentimento de alienação no local de trabalho (ISCI; TASTAN; AKYOL, 2013) e a intenção de abandonar o emprego (OZYILMAZ, 2010).

Para tanto, a conscientização dos enfermeiros sobre as características confiáveis de seus gestores também garante a identificação organizacional (TSENG; CHEN; CHEN, 2005), o desenvolvimento e a manutenção da confiança nos colegas estimulam os membros da equipe a cooperar, facilitando a comunicação interpessoal (BASIT; DUYGULU, 2018). E, ainda, enfermeiros que confiam em seus locais de trabalho foram vistos como mais propensos a mostrar bondade, honestidade e integridade (ALTUNTAS; BAYKAL, 20I0).

Um estudo foi realizado com a intenção de relacionar a confiança e o empoderamento no trabalho com 4I 2 enfermeiras canadenses (LASCHINGER; FINEGAN; SHAMIAN; WILK, 200I). Os resultados demostraram que enfermeiras empoderadas relataram uma maior confiança organizacional, o que, por sua vez, influenciou positivamente nas atitudes profissionais, no aumento do comprometimento afetivo e na satisfação no trabalho. 
Corroborando, outra pesquisa realizada na Austrália investigou a relação entre enfermeiros e seu gestor no engajamento e na confiança no trabalho relacionados a comportamentos discricionários e intenção de sair da organização (RODWELL; MCWILLIAMS; GULYAS, 20I 7). Os achados mostram que os aspectos de afeto e respeito foram positivamente relacionados à confiança. Já o engajamento e a confiança foram negativamente relacionados à intenção de sair da empresa, assim como a dimensão lealdade entre o enfermeiro e seu gestor

Nesse sentido, um ambiente confiável também pode induzir uma atmosfera de trabalho positivo, facilitando assim a união dos colegas de trabalho. Os empregados ficam mais satisfeitos quando trabalham em um ambiente agradável, onde possuem boas relações com os demais e acreditam que não estão sendo usados ou sabotados pela organização, em comparação com o trabalho em um ambiente desagradável e duvidoso (HSU; CHIANG; CHANG; HUANG; CHEN, 2015).

\section{PROCEDIMENTOS METODOLÓGICOS}

Para atingir o objetivo proposto de analisar a confiança de enfermeiros na organização hospitalar em que trabalham, optou-se por um estudo amostral, de abordagem quantitativa-qualitativa, descritiva e de corte transversal. A escolha de um método misto neste estudo se sustenta a partir da justificativa de que esse tipo de método promove o entendimento sobre o fenômeno de escolha de uma forma que não seria obtida com a utilização de somente uma abordagem. Ainda, a pesquisa adotou a estratégia de triangulação concomitante (CRESWELL; CLARK, 20I3) em que os dados qualitativos e quantitativos tiveram igual atribuição de peso e foram mixados mediante integração.

A amostra, não probabilística por conveniência, constituiu-se de 58 enfermeiros recrutados em um hospital localizado em uma cidade no norte do estado do Rio Grande do Sul. Os 58 enfermeiros fazem parte do quadro total de enfermeiros da organização hospitalar que trabalham no período diurno. Esses profissionais possuem mais de 90 dias de trabalho, ou seja, já passaram pelo contrato de experiência. Apenas não participaram do estudo os enfermeiros que estavam em licença saúde ou maternidade/paternidade e férias, isto é, que não se encontravam trabalhando no momento da pesquisa. Apesar do tamanho da amostra, que pode ser considerado pequeno, essa representa a totalidade do quadro de enfermeiros do hospital que presta serviço ao paciente, cerca de mais de $60 \%$ do quadro de profissionais, o que serve de justificativa para o tamanho amostral e sua relevância para análises estatística.

Optou-se por realizar a pesquisa neste hospital pela sua característica de grande porte, que atende as regiões norte, noroeste e central do Rio Grande do Sul e oeste de Santa Catarina. O hospital é a mais antiga instituição médico-hospitalar da cidade e tem como missão desenvolver e proporcionar assistência altamente qualificada para pessoas de todos os grupos sociais. Além disso, a instituição passa por uma fase de crescimento em que se destaca a edificação de novos prédios, a revitalização das áreas físicas já existentes, a implantação de novas tecnologias e os avanços no segmento de ensino e pesquisa.

A coleta de dados foi desenvolvida em dois momentos. Na primeira etapa, adotou-se a abordagem quantitativa, utilizando-se de uma escala de confiança a fim de coletar dados que possibilitam medir o quanto o empregado acredita que pode confiar na organização em que trabalha, realizada através da aplicação da Escala de Confiança do Empregado na Organização - ECEO (OLIVEIRA; TAMAYO, 2008). A ECEO em sua versão reduzida, contém 28 itens que fazem parte dos cinco fatores que antecedem a confiança na organização ((I) promoção do crescimento na organização; (2) solidez organizacional; (3) normas relativas à demissão do empregado; (4) reconhecimento financeiro organizacional; (5) padrões éticos) conforme exposto anteriormente. As questões objetivam medir o quanto o empregado acredita que pode confiar na organização em que trabalha. Os itens dessa escala foram respondidos com base em uma grandeza do tipo Likert de cinco pontos variando de I (discordo totalmente) a 5 (concordo totalmente.). Os fatores que compõem a ECEO bem como o número das questões da escala e o valor do Alfa de Cronbach - que mede a consistência interna sendo os valores < 
0,50 considerados inaceitáveis, 0,70 a 0,90, boa e 0,90 a I, muito boa (PEREIRA; PATRÍCIO, 20I3), são mostrados no Quadro 2.

Quadro 2-Fatores, número de itens, questões e alfa de Cronbach da ECEO

\begin{tabular}{|l|c|l|c|}
\hline Fatores & No de itens $^{\circ}$ & Questões & Alfa de Cronbach \\
\hline Promoção do crescimento do empregado & 5 & $7, \mathrm{I} 0, \mathrm{I} 2, \mathrm{I} 7, \mathrm{I} 9$ & 0,92 \\
\hline Solidez organizacional & 5 & $4, \mathrm{I} 3, \mathrm{I} 5, \mathrm{I}, 2 \mathrm{I}$ & 0,86 \\
\hline Normas relativas à demissão do empregado & 6 & $\mathrm{I}, 3,8, \mathrm{II}, \mathrm{I} 4,23$ & 0,79 \\
\hline Reconhecimento financeiro organizacional & 5 & $9, \mathrm{I} 6,22,25,28$ & 0,83 \\
\hline Padrões éticos & 7 & $2,5,6, \mathrm{I} 9,20,24,27$ & 0,93 \\
\hline
\end{tabular}

Fonte: Elaborado pelos autores (2020).

O cálculo do escore médio da ECEO é obtido somando-se os valores indicados pelo respondente e dividindo-se essa somatória pelo número de itens de cada fator definido. Dessa maneira, obtêm-se um valor que deverá situar-se entre um e cinco (OLIVEIRA; TAMAYO, 2008). Por exemplo, um valor igual a 4,2 no fator reconhecimento financeiro organizacional, demonstra que o respondente concorda que a organização reconhece por meio de incentivos financeiros seus empregados. Uma média muito baixa, por exemplo, I,7 no fator normas relativas à demissão do empregado, indica que o respondente desconhece ou discorda na sua totalidade, com as premissas desse contexto. Valores entre 4 e 5 são valores de concordância, entre I e 2,9 indicam discordância e valores entre 3 e 3,9 podem mostrar que o respondente está indiferente quanto ao fator analisado. A interpretação dos resultados obtidos deverá considerar que quanto maior for o valor do escore médio, mais o respondente demonstra sua concordância com o conteúdo avaliado por um dado fator.

$\mathrm{Na}$ segunda etapa, após todos terem respondido a ECEO, adotou-se uma abordagem qualitativa, buscando coletar dados, por meio do preenchimento de um questionário no formato de complementação de sentenças. O roteiro de entrevistas foi composto por cinco perguntas abertas, a fim de analisar os fatores que interferem na confiança dos enfermeiros. Nessa etapa, não foram entrevistados todos os enfermeiros, somente 8 profissionais participaram da complementação de sentenças e estão identificados com EI, E2... E8, para fins da análise dos dados, na próxima seção. Os 8 enfermeiros foram escolhidos aleatoriamente, conforme disponibilidade de participar do estudo e permaneceram na sala para finalizar a coleta dos dados. Os demais enfermeiros, foram liberados para voltar a seus postos de trabalho.

As sentenças foram organizadas a partir da ECEO e passadas por um pré-teste com 5 enfermeiros que não faziam parte da organização hospitalar estudada. As sentenças utilizadas foram: (I) Quanto à promoção do crescimento do empregado, posso afirmar que minha confiança no hospital...; (2) Quanto à solidez organizacional, posso afirmar que minha confiança no hospital...; (3) Quanto às normas relativas à demissão do empregado, posso afirmar que minha confiança no Hospital...; (4) Quanto ao reconhecimento financeiro organizacional, posso afirmar que minha confiança no hospital...; (5) Quanto aos padrões éticos, posso afirmar que minha confiança no hospital... .

Salienta-se que a parte inicial do questionário trazia informações gerais a respeito do estudo: o objetivo, a confiabilidade das respostas, as questões éticas, bem como orientações de preenchimento do questionário e acesso aos dados utilizados de modo sigiloso, somente para fins acadêmicos. O questionário foi desenvolvido em formato eletrônico e disponibilizado na plataforma Google Docs, composto por 40 questões em sua totalidade.

Ainda, por tratar-se de pesquisa que envolve seres humanos, o estudo foi encaminhado para apreciação do Comitê de Ética em Pesquisa da Faculdade Meridional (IMED). Esse procedimento está em conformidade com o disposto na Resolução 466/2012 do Conselho Nacional de Saúde (CONEP), sendo aprovado sob o número CAAE 49747215.2.0000.5319. Dessa forma, foram disponibilizados o Termo de Consentimento Livre e Esclarecido (TCLE) em duas vias aos enfermeiros que optaram em participar, sendo que o respondente ficou de posse de uma via, enquanto a outra permaneceu com os pesquisadores. O TCLE lhes assegura o sigilo dos dados coletados e o seu anonimato. Os dados coletados foram tabulados e organizados, primeiramente, em uma planilha do programa Microsoft Excel. 
Para a análise dos dados, as informações foram submetidas: (i) apuração dos resultados conforme procedimentos estabelecidos para a ECEO de acordo com Oliveira e Tamayo (2008) através da utilização do software Statistical Package for Social Science - SPSS (versão 2I), extraindo-se a média de cada item e de cada fator integrante da escala; (ii) análise do conteúdo (BARDIN, 2006) da complementação de sentenças, em categorias de dados, seguindo os cinco fatores nominados; e, (iii) descrição dos dados sociodemográficos dos enfermeiros. A seguir, são apresentados os resultados encontrados nesta investigação.

\section{ANÁLISE DOS RESULTADOS}

\section{Caracterização da amostra}

A amostra deste estudo constitui-se de 58 enfermeiros que atuam em um hospital localizado em uma cidade ao norte do Rio Grande do Sul. Primeiramente, realizou-se a conferência dos dados para verificar a existência de missing values (questões sem alguma(s) resposta(s)) e de outliers (respostas concentradas em uma única alternativa). Não houve exclusão de nenhum questionário por não apresentar missing values nem outliers, permanecendo, assim, a amostra inicial de 58. A caracterização da amostra encontra-se descrita na Tabela I, em que são representadas as questões de sexo, faixa etária, escolaridade, tempo de formação acadêmica e tempo de trabalho na organização hospitalar.

Tabela I- Caracterização sociodemográfica da amostra deste estudo $(\mathrm{n}=58)$

\begin{tabular}{ccc}
\hline Características & \% & $\mathbf{n}$ \\
Sexo & 87,8 & $5 \mathrm{I}$ \\
Feminino & I2,2 & 7 \\
Masculino & & \\
Faixa etária & 35 & 20 \\
22 a 32 anos & 47 & 27 \\
33 a 42 anos & 12 & 7 \\
43 a 52 anos & 6 & 4 \\
53 a 62 anos & & \\
Escolaridade & 18,2 & II \\
Graduação & 75,8 & 43 \\
Especialização & 6 & 4 \\
Mestrado/doutorado & & \\
Anos de graduado(a) & 47,2 & 27 \\
I a 5 anos & 23,5 & 13 \\
6 a I0 anos & 23,5 & 13 \\
II a 20 anos & 5,8 & 5 \\
2I a 30 anos & & \\
I a 5 anos & 50 & 29 \\
I a I0 anos & 20,7 & II \\
II a 20 anos & 23,5 & 13 \\
2I a 30 anos & 5,8 & 5 \\
\hline
\end{tabular}

Fonte: Desenvolvida pelos autores (2020).

A partir dos dados apresentados observa-se que a predominância dos respondentes do estudo é do sexo feminino $(\mathrm{n}=5 \mathrm{I} ; \%=87,8)$. Em relação à idade, 47\% $(\mathrm{n}=27)$ possuem entre 29 e 39 anos, sendo a idade média dos empregados de 35,58 anos. A amplitude foi de 34, variando de 22 a idade mínima e 56 a idade mais elevada. A escolaridade demonstra que além da graduação, a maioria dos respondentes $(n=43 ; \%=75,8)$ realizaram algum tipo de especialização. $O$ tempo de formação acadêmica, ou seja, da conclusão da graduação em enfermagem encontra-se na faixa de I a 5 anos $(n=27 ; \%=47,2)$ e o tempo 
que trabalham no hospital pesquisado para a maioria dos respondentes também está entre I e 5 anos $(\mathrm{n}=50 ; \%=29)$.

\section{Fatores que influenciam a confiança dos enfermeiros}

Evidenciou-se após a análise do Fator I (Tabela 2) que a média geral desse item foi de 3,I8, caracterizando indiferença dos respondentes quanto à concordância de que a organização hospitalar promove o crescimento do empregado. Nota-se que o item Q7 possui a média 3,49, a maior entre os itens pertencentes ao fator I e estando entre os valores de indiferença ( 3 e 3,9), e demonstrando que os empregados podem estar em desacordo com as políticas de incentivos ao crescimento ofertadas pela organização hospitalar. Tais resultados coadunam com as respostas obtidas por meio das perguntas fechadas (sentenças), quando mencionado que "até o momento desconheço programas de crescimento profissional que sejam formalmente divulgados" (E2). E ainda, quando dito que "mesmo com boas condições de trabalho deveriam incentivar mais o crescimento dos colaboradores" (E3).

Tabela 2 - Médias dos itens que compõem o fator I: "Promoção do crescimento do empregado na organização"

\begin{tabular}{l|c}
\hline Item & Média \\
\hline Q7- Esta organização oferece condições reais para que o empregado se desenvolva & 3,49 \\
\hline Q10 - Esta organização segue normas para promover seus empregados. & 3,2 I \\
\hline Q12 - Esta organização dá oportunidade de crescimento profissional ao empregado. & 3,38 \\
\hline Q17 - Esta organização incentiva o crescimento profissional de seus empregados. & 3,23 \\
\hline Q26 - O plano de carreira desta organização permite o crescimento profissional do empregado. & 2,62 \\
\hline Média geral fator I & 3,18 \\
\hline
\end{tabular}

Fonte: Elaborada pelos autores (2020).

Revela-se no estudo do Fator I que a organização, independentemente de ofertar condições de crescimento, não possui um plano de carreira satisfatório, visto que o item Q26, tratando especificamente deste assunto possui a pior média do fator, ou seja, 2,62, demonstrando a discordância dos empregados quanto à confiança na organização quando se refere ao plano de carreira.

Para Shane (2009), organizações com elevado crescimento, devem incentivar a formação de alta qualidade de seus empregados, proporcionando um crescimento exponencial em suas habilidades e competências. Outro estudo destaca a importância de que as pessoas se sintam realizadas e motivadas para que desempenhem bem suas funções (MELARA; BECCARIA; CARTA; CONTRIN, 2006). Ademais, acredita-se que esse aspecto possa impactar na cidadania organizacional (embora tal comportamento não seja reconhecido pelo sistema de recompensa formal) (CAVAZOTTE; HARTMAN; BAHIENSE, 2013), ele impacta no que se refere ao alcance de metas, o que de certa forma contribui para a efetividade organizacional (DIAS; OLIVEIRA, 20I6). Benefícios que podem ser representados pela satisfação dos clientes e o retorno nos percentuais de venda (RAUF, 20I4). Outro aspecto que sobre influência dessa variável é o clima organizacional, pois esse está relacionado a temática confiança (CHIUZI; SIQUEIRA; MARTINS, 20I2).

No Fator 2, que se refere a solidez organizacional, a questão Q13 possui a maior média entre os itens que compõem esse fator, revelando a concordância dos empregados quanto à posição de destaque que a organização hospitalar ocupa na área de saúde. Evidencia-se também que os empregados tendem a confiar na estabilidade financeira da organização, pois a média do item Q4 $(3,92)$ está muito próximo do índice de concordância da ECEO, estipulado a partir de Q4 (Tabela 3). Isso quer dizer que os empregados percebem que o hospital cumpre suas obrigações financeira, ou seja, o pagamento do salário pontualmente, e que a organização tem uma perspectiva de um futuro próspero, bem como a capacidade de superar crises econômicas ocasionadas por planos governamentais e mudanças de mercado. 
Tabela 3 - Médias dos itens que compõem o fator 2: "Solidez organizacional"

\begin{tabular}{l|c}
\hline Item & Média \\
\hline Q4 - Acredito na estabilidade financeira desta organização. & 3,92 \\
\hline Q13 - Esta organização ocupa uma posição segura na sua área de atuação. & 4,05 \\
\hline Q15 - Esta organização está preparada para sobreviver às crises econômicas. & 3,79 \\
\hline Q18 - A solidez econômica desta organização dá segurança aos empregados. & 3,59 \\
\hline Q2I - Esta organização é conhecida pelo seu poder econômico. & 3,33 \\
\hline Média geral fator 2 & 3,73 \\
\hline
\end{tabular}

Fonte: Elaborada pelos autores (2020).

Já a média geral do Fator $2(3,732)$ mostra que os funcionários estão indiferentes quanto à análise da solidez organizacional, porém o índice possui uma leve ascendência, chegando próximo a 4, revelando com isso um fato positivo quando comparado com os índices da ECEO, que variam de um a cinco. Esses resultados encontrados no fator que se refere à solidez organizacional podem também ser complementados quando mencionado:

Não temos atrasos nos salários e estamos todos juntos trabalhando para superar as crises econômicas. Sempre cumpre com suas obrigações em dia. Antecipa salário algumas vezes e outros benefícios. O hospital é uma empresa de princípios sólidos e nesse aspecto eu confio totalmente nele [no hospital] (EI).

Nesse contexto, preservar a solidez de uma organização advém de um processo de controle e monitoramento constante de seu desempenho, seguido da escolha da decisão mais adequada na solução de seus problemas (COSTA; NETO; ALVES, 2015). A reputação é um fator de extrema importância para a solidez de uma organização sendo vista como algo valoroso ou significativo para a organização, estando associada à noção de recursos ou ativos intangíveis, financeiros ou econômicos (ROSSONI; GUARIDO FILHO, 20I5). A capacidade de pagar os salários, de superar crises (OLIVEIRA; TAMAYO; 2008) e o próprio poder econômico representam a solidez da organização (VENTURA; TEIXEIRA; OLIVEIRA; PORTO, 2017), influenciando na percepção do empregado quanto a mesma Para Cunha et al. (2017), esse fator é relevante, pois encontra-se altamente relacionado ao comprometimento organizacional, elemento que está ligado ao baixo absenteísmo e à produtividade da organizacional.

No terceiro fator que refere-se às normas relativas à demissão do empregado, deduz-se após a crítica das médias dos itens constantes no Fator, que a discordância quanto às normas a respeito da demissão de empregados é geral entre os respondentes, havendo uma similaridade entre as médias do item QI (demissão sem explicação) e o item Q23 (demissão independente das normas) (Tabela 4). Revela-se com isso que o enfermeiro pode ser demitido sem saber o motivo, mesmo que tenha trabalhado dentro das normas da organização. Os demais itens configuram a discordância dos enfermeiros, conforme a ECEO.

Tabela 4-Médias dos itens que compõem o fator 3: "Normas relativas à demissão do empregado"

\begin{tabular}{l|c}
\hline Item & Média \\
\hline QI - Nesta organização, um empregado pode ser demitido sem receber explicações convincentes. & 2,28 \\
\hline Q3 - Esta organização considera apenas seus próprios interesses. & 2,49 \\
\hline Q8 - A demissão dos empregados segue um procedimento conhecido por todos. & 2,64 \\
\hline QII - Os dirigentes desta organização demitem empregados baseando-se em julgamentos pessoais. & $2,5 \mathrm{I}$ \\
\hline QI4 - As normas para demissão dos empregados são claras. & 2,59 \\
\hline Q23 - Aqui, os empregados são demitidos a qualquer momento, independente das normas da organização & 2,10 \\
\hline Média geral fator 3 & 2,43 \\
\hline
\end{tabular}

Fonte: Elaborada pelos autores (2020). 
A ausência de transparência nas normas demissionárias nas organizações pode ter relação na falta de satisfação, motivação, de envolvimento laboral e confiança na organização por parte do empregado (ARAÚJO; OLIVEIRA, 20I2). Clarificar as normas referentes à segurança profissional é uma forma do empregado avaliar o risco de demissão sem motivo, um meio de percebê-lo (KUBO et al., 2015). Nesse sentido, na complementação das sentenças, o E5 diz que "mesmo com as normas para demissão, muitas situações que a mesma deveria ocorrer não acontece dependendo de quem for o enfermeiro. São vários critérios adotados nas demissões. Fico insegura por momentos, não consigo confiar nesse aspecto.

Essas percepções representam um alerta sobre a situação atual do conhecimento pelos empregados ou da divulgação das normas a respeito de demissões da organização. Explicita a necessidade de identificação de um instrumento para divulgação de tais normas, com abrangência a todos os enfermeiros da organização hospitalar, conforme sugere o E6 "acredito que deveriam ser mais definidos estes critérios e ser mais enfatizado aos empregados". A pesquisa de Cunha et al. (2017), com 76 colaboradores de uma empresa, encontrou resultados divergentes, nela os participantes percebem a existência de normas referentes a demissão, o que permite a eles fazer uma previsão mais assertiva acerca de sua permanência na empresa. Os autores defendem que a percepção desses fatores em maior ou menor grau, por parte dos funcionários, contribui para aumentar a confiança do empregado na organização.

Por meio das respostas nas perguntas fechadas, não foi possível concluir qual o grau de confiança do enfermeiro quando instigado sobre as normas relativas às demissões, deduziu-se que esta parcela de respondentes desconhece as normas ou a sua aplicabilidade, podendo ser resultado de uma política de comunicação interna deficiente. Outrossim, exterioridades apontadas pelos enfermeiros como falta de autonomia para desligar subordinados, condutas desconhecidas para demissões por parte da organização, podem ser fatores que influenciaram nas justificativas quanto ao grau de confiança intraorganizacional, conforme dito que:

Apesar de não ter conhecimento de demissões com causa indefinida, também desconheço normas mais específicas a cerca de demissões. Sigo princípios profissionais gerais de minha profissão como: ética, comprometimento e responsabilidade. Assim, frente algumas condutas ficam à mercê de empregados, não demitido como deveria (E8).

Sendo assim, por ser uma parcela significativa dos respondentes, consolida-se a ideia da necessidade de uma ação da área de Recursos Humanos (RH) em prol da disseminação do conhecimento a respeito das políticas de desligamento entre os enfermeiros, visto que os enfermeiros também são responsáveis no processo demissionário de seus subordinados. Nesse sentido, o estudo de Holanda e Cunha (2005) contribui apresentando que, os hospitais admitem em sua maior parte enfermeiros com pouco tempo de formação e que estão em busca de continuar seu aprendizado teórico e consolidar habilidades, a fim de obter melhor prática e mais segurança para enfrentar um outro mercado de trabalho capaz de oferecer maiores benefícios, como plano de carreira. Assim, tal política de RH pode ser o fator contribuinte para um número significativo de desligamentos e para a ausência de uma política nesse aspecto, uma vez que, os maiores números de desligamento por parte dos enfermeiros são solicitados pelo empregado e não pelo empregador. Isso corrobora a amostra deste estudo, em que $50 \%$ dos enfermeiros possuem pouco tempo de casa e 47,2\% menos de 5 anos de formação em enfermagem.

No quarto fator que analisou o reconhecimento financeiro organizacional, analisando as médias dos itens integrantes do Fator 4, revela-se um quadro em que os enfermeiros nem concordam e nem discordam quanto ao reconhecimento financeiro por parte da organização (Tabela 5). Nota-se, porém, que o item Q28 com média de 3 ficou muito próximo do indicador de discordância dos enfermeiros, pois segundo a ECEO o limite é de 2,9.

Tabela 5 - Médias dos itens que compõem o fator 4: "Reconhecimento financeiro organizacional"

\begin{tabular}{l|c}
\hline Item & Média \\
\hline Q9 - O salário pago por esta organização corresponde aos esforços do empregado. & $3,5 \mathrm{I}$ \\
\hline Q16 - Os contratos estabelecidos por esta organização são vantajosos para todos. & 3,33
\end{tabular}


(Conclusão)

Q22 - Esta organização valoriza o trabalho do empregado financeiramente.

3,18

Q25 - Aumentar salário é uma forma de reconhecimento desta organização.

Q28 - O trabalho do empregado é reconhecido por esta organização por meio de salário.

Média geral fator 4

3,00

Fonte: Elaborada pelos autores (2020).

Evidencia-se, após a apreciação dos itens relativos ao reconhecimento financeiro, que esses merecem uma atenção especial por parte da organização. Conclui-se que o fator 4 é um elo fraco no construto confiança na organização. No entanto, existe compreensão por parte dos trabalhadores quanto ao salário que recebem, uma vez que "com o cenário econômico atual, é certo afirmar que a instituição gostaria de remunerar de uma melhor maneira, porém, frente ao mercado e a oferta, pode-se dizer que é satisfatório". E ainda, E8 complementa que "sabemos que o hospital caminha na medida das possibilidades concretas de execução".

Nesse contexto, a concessão de aumento salarial é percebida como uma das manifestações de reconhecimento por parte da organização. Tal reconhecimento financeiro traz benefícios para ambas as partes (ZANINI, 2007). Contudo, diversas são as formas de remuneração de pessoas nas organizações, entretanto, nas organizações hospitalares a forma mais comum é a fixa, ou seja, não há complementação variável nos salários recebidos (ZILLMER, 20I4). Esse aspecto demonstra descontentamento por parte de alguns enfermeiros, conforme evidenciado que "penso que deveria ter um abono para quem deseja se especializar e cumprimento de metas para devido aumento da remuneração”. E, também:

Acho que a instituição deve pensar em um programa salarial, beneficiando aqueles que buscam conhecimento, como por exemplo: especialização, mestrado, etc., ou seja, estimular a procura pelo conhecimento e ao mesmo tempo ser reconhecido financeiramente. Geralmente esta política só é adotada em instituições federais e municipais (E2).

Assim, nota-se por meio da análise das justificativas que alguns enfermeiros não estavam confiantes na organização quando o assunto tratado foi de ordem salarial. Os relatos deixaram transparecer que a organização carece de um plano de cargos e salários específico para a categoria, pois há o sentimento de não valorização para aqueles que buscam o crescimento profissional por meio de especializações ou cursos específicos da profissão. Tais achados vão ao oposto do estudo de Chiuzi, Siqueira e Martins (2012) que, ao estudarem 200 trabalhadores de empresas publicas e privadas, constataram, por meio da aplicação da ECEO que os trabalhadores percebem sua organização como justa no tocante à alocação de recursos para com eles e se sentem recompensados por seus esforços e sua experiência, acreditando que a organização é digna de sua confiança, sentindo-se satisfeitos com o salário que recebem.

Segundo Melara, Beccaria e Carta (2006), incentivar o empregado é um motivo externo que parte da política de recursos humanos de algumas empresas e representa o reconhecimento da administração pelo desempenho e esforço do trabalhador, sendo um destes o reconhecimento financeiro. Com a assinatura do contrato de trabalho entre organização e trabalhador, fica estipulado o seu valor de troca, ou seja, o salário, que pode ser considerado um símbolo de status para aqueles que o possuem (NEWSTROM; DAVIS, 200I).

Ainda, Van Busum e Mattke (2013) defendem que o incentivo financeiro se revela como um dos elementos-chave implementados pela organização ao praticar um conjunto de medidas que visam melhorar o bem-estar entre os empregados e a organização. Para os autores, recompensas simbólicas promovem a conscientização e o engajamento. Nesse sentido, torna-se claro, a necessidade de implantação de um plano de carreira, juntamente com um sistema de recompensas, com a finalidade de estimular a concorrência entre os enfermeiros e o consequente aumento de salário dos mesmos.

Parece não existir um elo entre a solidez organizacional, isso é, a mesma deveria se refletir no reconhecimento financeiro desejado pelos empregados. Isso que se verifica pelo fato de alguns não sentirem que seu desempenho está sendo devidamente remunerado e valorizado em termos de 
compensação financeira. Tal questão igualmente foi ponderada por Batista (2010), que, a organização que é percebida como capaz de garantir salários, benefícios e garantias ao trabalhador possui maior tendência de ser vista como sendo confiável e possuindo estabilidade financeira, o que garante segurança em relação ao trabalho. E ainda, corrobora com os achados de Horta, Demo e Roure (2012), segundo os autores, a política de recompensas pode ser tida como a melhor preditora de confiança do empregado na organizaçao.

Por fim, a análise das médias referentes ao Fator 5 - Padrões Éticos, revela uma proximidade a 4 quanto à média de concordância da ECEO. A média geral do fator 5 corrobora esta evidência. Fica claro também que os enfermeiros confiam na transparência da organização hospitalar quanto às informações prestadas aos seus clientes $(\mathrm{Q} 6=4,05)$, possuindo a maior médio entre os itens (Tabela 6).

Tabela 6 - Médias e desvios padrão dos itens que compõem o fator 5: "Padrões éticos"

\begin{tabular}{l|c}
\hline Item & Média \\
\hline Q2 - Esta organização é ética. & 3,84 \\
\hline Q5 - O empregado pode acreditar nas informações que esta organização divulga. & 3,97 \\
\hline Q6 - Para esta organização, o cliente tem o direito de ser informado sobre assuntos que lhe dizem respeito. & 4,05 \\
\hline Q19 - Os clientes desta organização sabem que podem acreditar na solidez dela. & 3,68 \\
\hline Q20 - Esta organização é conhecida por trabalhar de maneira responsável. & 3,97 \\
\hline Q24 - Ser honesta com os clientes é princípio ético desta organização. & 3,87 \\
\hline Q27 - O cliente é respeitado nesta organização. & 3,95 \\
\hline Média geral fator 5 & 3,89 \\
\hline
\end{tabular}

Fonte: Elaborada pelos autores (2020).

Deduz-se que os padrões éticos aplicados no hospital são reconhecidos pelos seus enfermeiros como um ponto forte na confiança na organização, conforme exposto que "a instituição sempre zela e capacita seu quadro funcional quanto a seguir e cumprir normas éticas" (E3). Bem como quando é referido que "a cultura institucional do hospital é de compromisso com a ética, honestidade e transparência" (EI). E, adicionalmente em "tenho confiança no serviço, senão não traria para cá a minha mãe" (E4).

Os mais variados e complexos problemas que envolvem o dia a dia da organização necessitam de parâmetros éticos balizem atuação dela tanto em relação aos seus concorrentes quanto aos seus empregados (BASTOS; YAMAMOTO; RODRIGUES, 20I3). Um princípio ético, transparência na divulgação das informações, deve ser de forma vertical, ou seja, da direção para os níveis subordinados; e horizontal, entre os empregados de mesmo nível de subordinação (SILVA, 2013). Nesse aspecto, E7 comenta que "sempre comunicam e solicitam trabalho com qualidade, dentro das regras e normas".

São os padrões éticos que orientam a relação da organização com seus clientes e empregados ao procurar não prejudicar aqueles com os quais se relaciona. Para Sarmento (2008), o código de ética já é uma constante na quase totalidade das empresas, independente de porte, tornando-se esse o caminho para construção de qualquer programa de ética organizacional. Nesse ponto, E3 reforça que "seguimos sempre o protocolo da instituição". Nesse aspecto, quando os empregados associam ou se identificam com os objetivos, normas, valores e crenças da organização, é mais provável que atribuam níveis mais altos de confiança à organização (WILLIAMS, 2005; IANAGUIVARA, 20I I).

Quanto aos padrões éticos, essa variável está relacionada a um maior ou menor grau de confiança do empregado na organização (CUNHA et al., 20I7). Além disso, Dias e Oliveira (20I6), identificaram que a confiança na ética é um preditor significativo de sugestões criativas ao sistema e de clima favorável, ao indicar que a confiança nos padrões éticos da organização reflete na emissão de sugestões e de novas ideias para a organização, e, também, na divulgação de seus benefícios para pessoas externas a mesma.

Verifica-se alta concordância entre os participantes quanto a solidez da organização e os seus padrões éticos. O que se pode concluir, ante ao exposto é que os empregados trabalham mais satisfeitos em ambientes em que acreditam não estarem sendo usados ou sabotados pela organização, quando comparado a ambientes duvidosos (HSU; CHIANG; CHANG; HUANG; CHEN, 20I5). 


\section{CONSIDERAÇÕES FINAIS}

Esta pesquisa teve como objetivo analisar a confiança de enfermeiros dentro do hospital em que trabalham. Buscou-se quantificar a confiança dos enfermeiros na organização, evidenciando as questões que interferem nessa relação, através dos cinco fatores que fazem parte da Escala de Confiança Organizacional do Empregado - ECOE e qualitativamente, através da complementação de sentenças, que buscou por meio de perguntas fechadas as percepções de confiança dos enfermeiros perante a organização.

Após a análise dos dados obtidos pela ECEO e pela complementação de sentenças, nota-se que o Fator 5 (padrões éticos) foi a dimensão que apresentou maior nível confiança por parte dos enfermeiros perante o hospital. Apesar do valor encontrado estar na média geral de 3,89, este valor foi o mais elevado dentre as cinco dimensões da ECEO, estando muito próximo do valor de concordância total da escala (entre 4 e 5). Isso demonstra que os profissionais observam e confiam nos princípios estabelecidos no hospital, como a honestidade, a transparência, o respeito e a responsabilidade da organização. Tais achados também se comprovam com a complementação das sentenças em que os enfermeiros demonstraram confiar nos padrões éticos organizacionais.

Por outro lado, os resultados demonstraram uma discordância por parte dos enfermeiros nas questões ligadas as normas relativas a demissão do empregado (Fator 3 ), uma vez que, a média geral dessa dimensão foi de 2,43. Nesse sentido, os respondentes não reconhecem a existência de regras que orientam a organização a tomar decisões sobre demissão de empregados, o que poderá fazer com que não enxerguem previsões assertivas acerca de sua permanência na organização, uma vez que, não existem normas claras quanto ao desligamento dos enfermeiros. Esse resultado poderá auxiliar os dirigentes da organização hospitalar a criarem mecanismos de melhorias nessa questão, tendo em vista que, a falta de confiança no trabalho pode levar rapidamente o grupo a condição de falência social, em que as relações entre empregado-organização se apresentam altamente comprometidas pelas variáveis da confiança. Sem contar que essa dimensão pode estar relacionada ao absenté́smo, perda da criatividade e ao aumento da rotatividade.

Os demais fatores (promoção do crescimento do empregado; solidez organizacional e reconhecimento financeiro organizacional), apesar de apresentarem suas médias gerais classificadas como indiferentes pela ECEO, percebe-se com a análise da complementação de sentenças que os enfermeiros demonstram estarem confiantes (em partes) nesses aspectos. Os enfermeiros concordam que o hospital estimula a promoção e o crescimento interno, salvo discordância por não possui um plano de carreira satisfatório, motivo de descontentamento do grupo. Ainda, concordam que o hospital é uma empresa de princípios sólidos e transparente, bem como, são reconhecidos financeiramente, havendo apenas discordância quanto a falta de um incentivo financeiro àqueles que buscam melhores qualificações, não havendo distinção salarial de um enfermeiro que tem graduação com um que fez mestrado/doutorado, por exemplo.

Nesse contexto, os resultados dessa pesquisa oferecem subsídios para adoção de medidas gerenciais estratégicas e com referência ao comportamento organizacional, visto que contempla uma gama de dados importantes quanto à análise da confiança dos enfermeiros na organização. Pode-se levar o mesmo modelo de pesquisa a todos os setores da organização em separado ou de uma forma única com todos os empregados envolvidos em um só estudo. Assim, os resultados alcançados neste estudo mostram caminhos a serem percorridos nas pesquisas sobre confiança organizacional e enfermeiros. É nítida a carência de estudos a respeito da interface entre os temas aqui estudados. Assim, esta pesquisa aponta a necessidade de conhecer e investir em variáveis que antecedam a confiança do empregado na organização, tendo em vista que, já que esse é um fator que oferece melhoria da interação empregado-organização e que tem capacidade para influenciar outras instâncias no contexto organizacional, como a performance e a produtividade.

As descobertas relativas aos fenômenos estudados acrescentam conhecimentos na construção do entendimento sobre a confiança organizacional, uma vez que, os parâmetros utilizados para sua mensuração desse constructo foram construídos sobre dados empíricos, fundamentados em uma literatura ampla e consistente. Pode-se considerar que esta pesquisa oferta à literatura um acréscimo ao estudo das 
relações de confiança entre indivíduo e organização, relatando pontos fortes e de melhorias quanto ao seu trato relacional. E, ainda, contribui para novas investigações a respeito das relações de confiança, principalmente quando tratada dentro de organizações hospitalares, visto que foi constatada uma insuficiência no tocante à literatura, principalmente nacional, conforme mencionado anteriormente. Adicionalmente com relação as contribuições deste estudo, há indicações de possa auxiliar na formulação de ações que possibilitem, auxiliar na promoção e manutenção da saúde do trabalhador, afetando consequentemente a produtividade institucional. Observa-se a necessidade de desenvolver estratégias diferentes para a medida da confiança organizacional.

Ainda, o questionário utilizado neste estudo pode colaborar na construção de ferramentas para planejamento e gestão estratégica em organizações de todos os portes e segmentos e podem ser direcionados aos empregados em geral e não a uma profissão ou cargo específico. Embora o estudo tenha seguido uma sequência metodológica, fica exposta sua limitação quanto ao número reduzido de respondentes, o que requer cuidado para generalização do estudo.

Por fim, sugere-se estudos futuros mais aprofundados em questionamentos, como: A confiança organizacional é influenciada pelo tempo de empresa ou o tempo de permanência na organização é influenciado pela confiança construída? A relação de confiança versus tempo de permanência na organização é biunívoca? Ainda, recomenda-se outros estudos com o acréscimo de variáveis à analise da confiança, como o suporte organizacional e o bem-estar de forma que represente, assim, uma contribuição teórica e prática para o campo de estudo do comportamento organizacional.

\section{REFERENNCIAS}

AKGUNDUZ, Y.; GUZEL, T. The effect of organizational trust as a mediator role on the relationship between organizational justice with organizational commitment. Journal of Social Sciences, v. I4, n. 3, p. I-I8, 2014.

ALTUNTAS, S.; BAYKAL U. Relationship between nurses' organizational trust levels and their organizational citizenship behavior's. Journal of Nursing Scholarship, v. 42, n. 2, p. I86-194, 2010.

ANDRADE, T.; ESTIVALETE, V.; COSTA, V.; FALLER, L.; COSTA, G. Interações entre os comportamentos de cidadania organizacional, confiança interpessoal, confiança organizacional e suporte organizacional. Revista de Administração da UFSM, v. II, edição especial, p. 489-504, 2018.

ARAÚJO, P.; OLIVEIRA, A. Bem-estar no trabalho: impacto das percepções dos valores dos valores organizacionais e da confiança do empregado na organização. In: Programa Institucional de Apoio à Iniciação Científica, I, 20I2, Uberlândia. Anais... Uberlândia: Universidade Federal de Uberlândia, 2012.

BARDIN, L. Análise de conteúdo. Lisboa: Edições 70, 2006.

BARROS, M. Inteligência emocional, confiança do empregado na organização e bem-estar no trabalho: um estudo com executivos. 20I I. 98 f. Dissertação (Mestrado em Psicologia da saúde). Universidade Metodista de São Paulo, São Bernardo do Campo - SP, 201 I.

BASIT, G.; DUYGULU, S. Nurses' organizational trust and intention to continue working at hospitals in Turkey. Collegian, v. 25, n. 2, p. I63-169, 2018.

BASTOS, A.; YAMAMOTO, O.; RODRIGUES, A. Compromisso social e ético: desafios para a atuação em psicologia organizacional e do trabalho. Porto Alegre: Artmed, 2013. 
BATISTA, R. Percepção de suporte organizacional, afeto positivo, afeto negativo e resiliência: Antecedentes da confiança do empregado na organização. 20I0. I45 f. Dissertação (Mestrado em Psicologia Aplicada). Instituto de Psicologia, Programa de Pós-graduação em Psicologia, Universidade Federal de Uberlândia - Uberlândia, 2010.

BATISTA, R.; OLIVEIRA, A. Antecedentes da confiança do empregado na organização. Estudos de Psicologia, v. I7, n. 2, p. 247-254, 2012.

BOE, T. Gaining and/or maintaining employee trust within service organizations. 2002. 9I s. Thesis (Master of Science Degree in Training and Development). University of Wisconsin, Madison, 2002.

CAVAZOTTE, F.; HARTMAN, N. S.; BAHIENSE, E. Charismatic leadership, citizenship behaviors, and power distance orientation: Comparing Brazilian and U.S. Workers. Cross-Cultural Research, v. 48, n. I, p. 3-3I, 2013.

CHHETRI, P. The role of cognitive and affective trust in the relationship between organizational justice and organizational citizenship behavior: A conceptual framework. Business: Theory and Practice, v. I5, n. 2, p. I70-I78, 2014.

CHIUZI, R.; SIQUEIRA, M.; MARTINS, M. As dimensões da organização positiva e seus impactos sobre o bem-estar dos trabalhadores. Mudanças - Psicologia da Saúde, v. 20, n. I-2, p. 3I-40, 2012.

COSTA, S.; NETO, J.; ALVES, K. A importância do profissional de controladoria e finanças para evitar desconsideração da personalidade jurídica em caso de violação ao princípio da entidade contábil. Revista Científica Facped, v. 5 n. I, p. I4-22, 2015.

CRESWELL, J.; CLARK, V. Pesquisa de métodos mistos. 2. ed. Porto Alegre: Penso, 2013.

CUNHA, D.; SILVA, A.; ESTIVALETE, V.; HÖRBE, G. Confiança do empregado na organização e comprometimento organizacional: em busca da relação entre os construtos. Sistemas \& Gestão, v. I2, n. I, 2017.

DECONINCK, J. The effect of organizational justice, perceived organizational support, and perceived supervisor support on marketing. Journal of Business Research, v. 63, s/n, p. I349-I355, 2010.

DESMET, P.; CREMER, D.; VAN DIJK, E. Trust recovery following voluntary or forced financial compensations in the trust game: The role of trait forgiveness. Personality and Individual Differences, v. 5I, n. 3, p. 267-273, 20II.

DIAS, F.; OLIVEIRA, Á. Valores e confiança organizacionais: fatores determinantes nos comportamentos de civismo organizacional. Temas em Psicologia, v. 24, n. 3, p. I087-I I00, 2016.

FISHER, R.; NOVELLI, J. Confiança como fator de redução da vulnerabilidade humana no ambiente de trabalho. RAE - Revista de Administração de Empresas, v. 48, n. 2, 2008.

FULMER, C.; GELFAND, M. Trust after violations: Are collectivists more or less forgiving? Journal of Trust Research, v. 5, n. 2, p. I09-I3I, 2015.

GILLESPIE, N.; DIETZ, G. Trust repair after an organization level failure. Academy of Management Review, v. 34, n. I, p. I27-I45, 2009. 
GOERGEN, C.; PAULI, J.; CERUTTI, P.; PERIN, M. The organizations. Justice, trust and retaliatory attitude: a study in a company in the metal-mechanic sector. Cuadernos de Administración, v. 34, n. 62, p. 33-49, 2019.

HOLANDA, L.; CUNHA, I. Tempo de permanência de enfermeiros em um hospital-escola e valores monetários despendidos nos processos de admissão, desligamento e provimento de novo profissional. Revista Latino-Americana de Enfermagem, v. I3, n. 5, p. 642-647, 2005.

HORTA, P.; DEMO, G.; ROURE, P. Políticas de gestão de pessoas, confiança e bem-estar: estudo em uma multinacional. Revista de Administração Contemporânea, v. I6, n. 4, p. 566-585, 2012.

HSU, C.; CHIANG, C.; CHANG, C.; HUANG, H.; CHEN C. Enhancing the commitment of nurses to the organisation by means of trust and monetary reward. Journal of Nursing Management, v. 23, s/n, p. 567-576, 2015.

IANAGUIVARA, C. Confiança do empregado na organização: Revalidação de instrumento de medida. 20II. I03 f. Dissertação (Mestrado em Ciências Humanas). Instituto de Psicologia, Universidade Federal de Uberlândia - MG - Brasil, $201 \mathrm{I}$.

ISCAN, O.; SAYIN, U. Orgutsel adalet, is tatmini ve orgutsel guven arasindaki iliski. Ataturk Universitesi Iktisadi ve Idari Bilimler Dergisi, v. 24, n. 4, p. I95-216, 2010.

ISCI, E.; TASTAN, S.; AKYOL, C. The role of managerial communication skills on the effect of organizational trust on job alienation: A case of private hospital employees. International Refereed Journal of Humanities and Academic Sciences, v. 2, n. 3, p. 95-II3, 2013.

KIM, T.; WANG, J.; CHEN, J. Mutual trust between leader and subordinate and employee. Journal of Business Ethics, v. I49, n. 4, p. 945-958, 2016.

KUBO, E.; CASTRO, D.; NUNES, V.; THIAGO, F. Frankenstein and organizational climate: Bibliographic study on an indefinite construct. Revista Internacional de Investigación En Ciencias Sociales, v. II, n. I, p. 35-54, 2015.

LASCHINGER, H.; FINEGAN, J.; SHAMIAN, J.; WILK P. Impact of structural and psychological empowerment on job strain in nursing work settings: expanding Kanters model. Journal of Nursing Administration, v. 3I, n. 5, p. 260-272, 2001.

LOURENÇO, C.; PINTO, A.; PEREIRA, C.; FONSECA, C.; NUNES, I.; ALMEIDA, M.; MENDES, O.; TOLLETI, G.; LOPES, M.; GÂNDARA, M. Confiança versus desconfiança na relação de cuidar: confiança enfermeiro-cliente, um conceito em construção no CHLN-HPV. Pensar Enfermagem, v. I5, n. 2, p. 3-I3, 201 I.

LUIS, P. P.; RODRÍGUEZ, N. D.; GÓMEZ, J. Á. R. R'ṣ̌pi ‘Existe una buena relación entre el personal de enfermeria y la organización sanitaria en la que trabajan? Un enfoque psicossocial. ENE - Revista de Enfermeria, v.I3, n. 3, p. I-I6, 2020

MAGUIRE, S.; PHILLIPS, N. 'Citibankers' at Citigroup: A study of the loss of trust after a merger. Journal of Management Studies, v. 45, n. 2, p. 372-401, 2008.

MELARA, S.; BECCARIA, L.; CARTA, A.; CONTRIN, L. Motivation of the nursing team in Intensive Care Unit. Health Sciences Files, v. I3, n. 3, p. 6I-70, 2006. 
NEVES, P.; CAETANO, A. Social exchange processes in organizational change: The roles of trust and control. Journal of Change Management, v. 6, n. 4, p. 35I-364, 2006.

NEWSTROM, J.; DAVIS, K. Comportamento humano no trabalho: Uma abordagem organizacional. São Paulo: Pioneira Thomson, 200I.

NIENABER, A.; HOFEDITZ, M.; SEARLE, R. Do we bank on regulation or reputation? A metaanalysis and meta-regression of organizational trust in the financial services sector. International Journal of Bank Marketing, v. 32, n. 5, p. 367-407, 2014.

OLIVEIRA, A. Confiança do empregado na organização: impacto dos valores pessoais, organizacionais e da justiça organizacional. Tese (Doutorado em Psicologia). 2004. Programa de Pós-graduação em Psicologia, Universidade de Brasília, Brasília - DF, 2004.

OLIVEIRA, Á.; SOUZA, M. Confiança do empregado na organização: o impacto dos valores pessoais e organizacionais. Revista Psicologia: Organizações e Trabalho, v. I4, n. 2, 2014.

OLIVEIRA, A.; TAMAYO, A. Confiança do empregado na organização. In: SIQUEIRA, M. (Ed.), Medidas do comportamento organizacional: Ferramentas de diagnóstico e gestão. Porto Alegre: Artmed, 2008.

OZARAS, G.; ABAAN, S. Investigation of the trust status of the nurse-patient relationship. Nursing Ethics, v. 25, n. 5, p. 628-639, 2016.

ÖZYILMAZ, Y. Vertical trust in organizations: a review of empirical studies over the last decade. Journal of Social Sciences Institute, v. 7, n. I3, p. I-28, 2010.

PEREIRA, A.; PATRÍCIO, T. Guia prático de utilização do SPSS: Análise de dados para ciências sociais e psicologia. 8. ed. Lisboa: Edições Sílabo, 2013.

RAUF, F. Perception of organizational justice as a predictor of organizational citizen- ship behavior: An empirical study at schools in Sri Lanka. European Journal of Business and Management, v. 6, n. I2, p. I24-I30, 2014.

RHEE, K. 'Different effects of workers' trust on work stress, perceived stress, stress reaction, and job satisfaction between Korean and Japanese workers. Safety and Health at Work, v. I, n. I, p. 87-97, 2010.

RODWELL, J.; MCWILLIAMS, J.; GULYAS, A. The impact of characteristics of nurses' relationships with their supervisor, engagement and trust, on performance behaviours and intent to quit. Journal of Advanced Nursing, v. 73, n. I, p. 190-200, 2017.

ROSSONI, L.; GUARIDO FILHO, E. O que faz um nome? Status, conselho de administração e características organizacionais como antecedentes da reputação corporativa. Revista de Administração RAUSP, v. 50, n. 3, p. 292-309, 2015.

RUEDA, F.; SERENINI, A.; MEIRELES, E. Relação entre qualidade de vida no trabalho e confiança do empregado na organização. Revista Psicologia Organizações e Trabalho, v. I4, n. 3, p. 303-3I4, 2014. 
SAMPAIO, T.; GOMIDE Jr, S.; OLIVEIRA, Á. Bem-estar no trabalho, gestão e justiça no trabalho em modelo mediacional. Revista Laborativa, v.8, n. I, p. 26-43, 2019.

SARMENTO, A. Códigos de ética empresarial: uma análise de fatores que influenciam a efetividade. 2008. 84 F. Dissertação (Mestrado em Administração e Desenvolvimento Empresarial). Departamento de Pós-graduação em Administração e desenvolvimento Empresarial. Universidade Estácio de Sá, Rio de Janeiro - RJ, 2008.

SEARLE, R.; DEN HARTOG, D.; WEIBE, A.; GILLESPIE, N.; SIX, N.; HATZAKIS, T.; SKINNER, D. Trust in employer: the role of high-involment work practices and procedural justice in European organizations. The International Journal of Human Resource Management, v. 22, n. 5, p. I069-I092, 2011.

SHANE, S. Why encouraging more people to become entrepreneurs is bad public policy? Small Business Economics, v. 33, s/n, p.I4I-I49, 2009.

SILVA, J. A comunicação interna nas instituições de ensino superior: um estudo comparativo entre duas IES do DF. 2013. 70 f. Trabalho de Conclusão de Curso (Pós-graduação em Gestão da Comunicação nas Organizações). Instituto CEUB de Pesquisa e Desenvolvimento - ICPD. Curso de Gestão da Comunicação, Centro Universitário de Brasília, Brasília - DF, 2013.

SILVA, U.; OLIVEIRA, Á. Qualidade de vida e valores nas organizaçoes: Impactos na confiança do empregado. Psicologia: Ciência e Profissao, v. 37, n. I, p. 7-I7, 2017.

SOLOMON, R.; FLORES, F. Construa confiança. Rio de Janeiro: Record, 2002.

TAN, H.; LIM, A. Trust in co-workers and trust in organizations. The Journal of Psychology, v. I43, n. I, p. 45-66, 2009.

TAN, H.; TAN, C. Toward the differentiation of trust in supervisor and trust in organization. Genetic, Social, and General Psychology Monographs, v. I26, n. 2, p. 24I-260, 2000.

TOP, M.; TEKINGUNDUZ, S. The effect of perceived organizational justice on job satisfaction and burnout levels of hemodialysis nurses, Journal of Renal Care, v. 45, n. 2, p. I20-I28, 2019.

TSENG, H.; CHEN, T.; CHEN, F. Constructing and testing a model of trustworthiness, trust behaviour and organizational identification. The Journal of Nursing Research, v. I3, s/n, p. 293-330, 2005.

TZAFRIR, S.; HAREL G. Trust-me: a scale form measuring manager employer trust worthiness. Academy of Management Review, v. 2, n. 2, p. II5-I32, 2002.

VAN BUSUM, K.; MATTKE, S. Financial incentives: only one piece of the workplace wellness puzzle comment on "corporate wellness programs: implementation challenges in the modern american workplace”. International Journal Health Policy Management, v. I, n. 4, p. 3I I-3I2, 2013. VENTURA, E.; TEIXEIRA, L.; OLIVEIRA, R.; PORTO, L. Assédio moral e seu dano na saúde mental dos indivíduos. RAHIS - Revista de Administração Hospitalar e Inovação em Saúde, v. I4, n. 4, 2017.

VELEZ, P.; STROM, T. Effects of organizational trust. Organization Development Journal, v. $30 \mathrm{n}$. 2, p. 39-50, 2012. 
VERBURG, R.; NIENABER, A.; SEARLE, R.; WEIBEL, A.; DEN HARTOG, D.; RUPP, D. The role of organizational control systems in employees' organizational trust and performance outcomes. Group \& Organization Management, v. 43, s/n, p. I79-206, 2018.

WILLIAMS, L. Impact of nurses' job satisfaction on organizational trust. Health Care Management Review, v. 30, n. 3, p. 203-2II, 2005.

ZANINI, M. Confiança interorganizacional como chave para a coordenação implícita e para a construção do capital intangível. Cadernos EBAPE, v. 5, n. 3, p. I-I3, 2007.

ZILLMER, M. A gestão da renumeração: o caso de uma Concessionária de Energia Elétrica do RS. Trabalho de Conclusão de Curso (Pós-graduação em Gestão de Pessoas). 20I4. I6 f. Departamento de Ciências Administrativas, Contábeis, Econômicas e da Comunicação. Universidade Regional do Noroeste do Estado do RS - Unijuí, Ijuí - RS. 